Revista Sostenibilidad, Tecnología y Humanismo

44

https://doi.org/10.25213/2216-1872.34

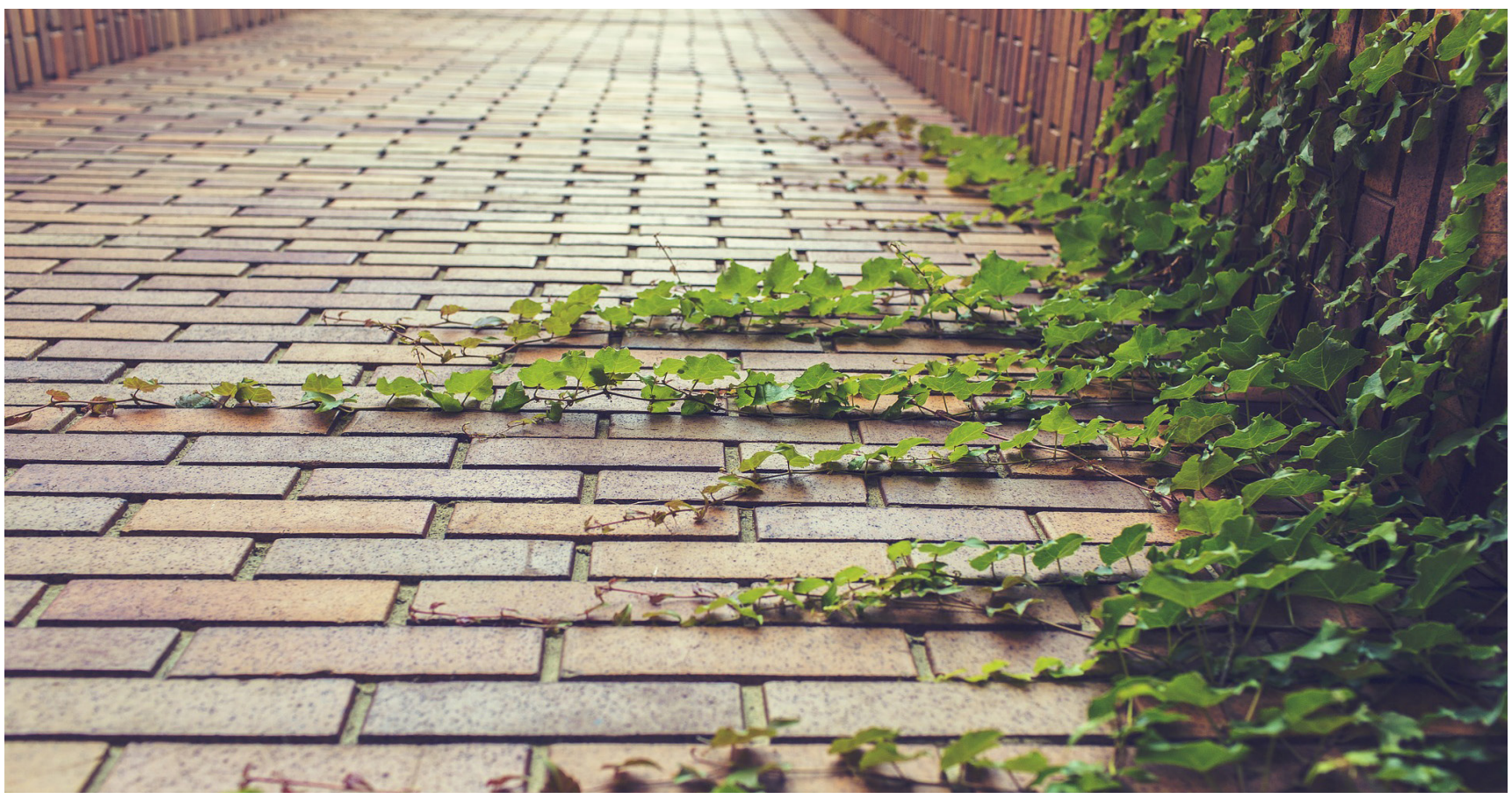

\title{
Propuesta de adoquines hechos a base de caucho reciclado
}

\section{Proposal for pavers made of recycled rubbeí}

Luz Angil Ramírez-Pico

Estudiante de Ingeniería Civil, Universidad Francisco de Paula Santander, Cúcuta, Colombia

Andres Esteban Orjuela-Rodríguez

Estudiante de Ingeniería Civil, Universidad Francisco de Paula Santander, Cúcuta, Colombia

Gina Ester Angulo-Blanquisett

Ingeniero Civil, gina.angulo@utadeo.edu.co,

https://orcid.org/0000-0001-5020-1933, Universidad Jorge Tadeo Lozano,

Cartagena de Indias, Colombia 
Cómo citar: L.A Ramirez-Pico, A.E Orjuela-Rodriguez y G.E Angulo-Blanquisett, "Propuesta de adoquines hechos a base de caucho reciclado", Sostenibilidad, Tecnología y Humanismo, vol. 11, no. 1, 44-53, 2020

\section{Resumen}

Los adoquines se han utilizado desde épocas remotas como ayuda a la estabilización de pisos, los primeros utilizados fueron las mismas piedras cubriendo los terrenos para unificar su recubrimiento por algo más estable. Actualmente esto ha evolucionado y pasado a niveles industrializados para la fabricación de este material, es por ello que el propósito de este trabajo de investigación es diseñar adoquines con base de fabricación de caucho reciclado, y así poder reducir el impacto que tiene este material sobre el ambiente, lo que se espera de esta propuesta que se basa en una metodología cualitativa con base a un diseño descriptivo es poder mejorar esos impactos ambientales y a su vez manejar un material más accesible y económico a poblaciones de bajos recursos y apoyar la trama urbana de esos contextos.

Palabras clave: Adoquines, suelos, caucho, reciclaje.

\section{Abstract}

Paving stones have been used since ancient times as an aid to the stabilization of floors, the first ones used were the same stones covering the land to unify its covering with something more stable. Currently this has evolved and passed to industrialized levels for the manufacture of this material, that is why the purpose of this research work is to design paving stones based on the manufacture of recycled rubber, and thus be able to reduce the impact that this material has on the environment, what is expected of this proposal that is based on a qualitative methodology based on a descriptive design is to be able to improve these environmental risks and in turn handle a more accessible and economic material to lowincome populations and support the urban fabric of those contexts.

Key words: cobblestones, floors, rubber, recycling. 


\section{6}

Introducción

Las basuras y desechos se están convirtiendo en el mayor problema para las grandes civilizaciones, el acumulamiento de los residuos ha ido evolucionando y hoy en día se encuentra en un estado crítico debido al gran volumen de desechos generados a diario.

Antiguamente en muchas ciudades los desechos simplemente se dejaban en el suelo de las casas o se arrojaban a la calle, lo que eventualmente causaba que el nivel de las calles subiera. En la actualidad, por ejemplo, la ciudad inglesa de Bath se encuentra de tres a seis metros más arriba que en tiempos del imperio romano [1]. Uno de los mayores residuos generados anualmente es el caucho ya que en 2014, las estadísticas consolidadas sobre el mercado del caucho, el consumo mundial de esta materia prima fue de 28,9 millones de toneladas [2].

Así mismo, en Colombia las basuras se están convirtiendo en una bomba de tiempo pues en el año 2015 Colombia generó alrededor de casi 10 millones de toneladas de los cuales el $96.8 \%$ fueron a parar a rellenos sanitarios [3]. Esto es una alarma pues en Colombia se cuenta con alrededor de 147 rellenos sanitarios de los cuales 13 tienen licencia vendida y muchos más ya tienen pocos años de vida útil.

En Colombia el reciclaje del caucho está creciendo poco a poco pues con caucho, además del asfalto modificado, se puede utilizar granulado para canchas sintéticas, calzado, adoquines, como sustituto del aserrín y para reemplazar combustibles fósiles tradicionales, pues, según expertos consultados, llega a generar mayor energía que el carbón. Con la aplicación de tecnologías físico-químicas también se pueden obtener otros subproductos, como aceites y combustibles tipo diésel, tras ser refinado [4]. En Cúcuta, Norte de Santander una persona en promedio genera un kilo de desechos al día lo que se traduce en un total de 710 toneladas de desecho solido diarias, pero ¿qué tanto de estos desechos son reciclados, pues en la ciudad solo se recicla alrededor del $6 \%$ de estos desechos, los materiales más reciclados son el vidrio seguido por el aluminio, cartón y plástico. Los desechos están siendo aprovechados para construir materias primas para parques infantiles, canchas sintéticas, muebles, láminas para edificar casas, etc [5], por un lado se reduce la contaminación generada por los residuos de los sectores productivos, con el aprovechamiento de los subproductos siendo posible mejorar ciertas propiedades tecnológicas de estos materiales para uso en la construcción [6].

De igual forma el negocio de reciclaje se ha convertido en una gran oportunidad para emprender pues según algunos emprendedores locales con materia reciclada se pueden hacer infinidad de cosas, como por ejemplo jardines o casas a base de materiales plásticos entre otras cosas. Los que resulta beneficioso para el medio ambiente y la concientización de las personas al reciclaje [7]. La falta de conciencia ecológica en los ciudadanos de Cúcuta al desechar las llantas de caucho y darle poca importancia al reciclaje y reutilización del caucho. Con este fin partimos de la idea del diseño de un adoquín hecho con caucho reciclado que cumpla con las normas mínimas de construcción de vías terciarias y senderos peatonales.

La mayoría de personas realizan un mal manejo de productos quien aún tienen vida útil, como por ejemplo tenemos el desecho de las llantas de vehículos, que en muchos casos son arrinconados por considerarse como objetos inservibles, convirtiéndose en criaderos de animales e inclusive causando enfermedades, es por ello que con este proyecto se busca que las personas reutilicen las llantas en forma útil y amigable con el medio ambiente. 
El presente trabajo de investigación tiene como finalidad obtener una dosificación estimada de un adoquín construido principalmente de caucho reciclado con el fin de contribuir a la construcción sostenible y la disminución de las basuras de caucho que diariamente son quemadas o tiradas, fomentar el reciclaje en la ciudad dando lugar a plazas de trabajo para recicladores, etc.

Se espera obtener un producto terminado listo para la comercialización con múltiples beneficios en el ahorro del tiempo de construcción de vías y caminos peatonales teniendo características personalizadas como el color o su forma, además de obtener un beneficio económico debido al bajo costo de la materia prima y la facilidad de obtenerla también se espera obtener información sobre el caucho como un elemento constructivo dando a conocer los porcentajes y dosificaciones ideales para el uso del caucho en este tipo de construcción.

El aporte teórico de la investigación es dar a conocer el comportamiento del caucho reciclado como material de construcción para vías y pasos peatonales, demostrar sus capacidades físicas y su resistencia en diferentes fuerzas actuantes, por ello el alcance de está encaminado a proponer la integración del caucho a un adoquín como forma de reciclado.

\section{Estado del arte}

A nivel nacional se analizaron trabajos para así aportar algunos puntos de vista a la presente con base en lo analizado. En ese sentido [8] en la investigación tuvo como objetivo la elaboración de un adoquín con concreto sustituyendo parte del agregado fino por polvillo de caucho proveniente de neumáticos reciclados en diferentes proporciones con el fin de cumplir con las normas establecidas, también se tuvo como objetivo la construcción de un adoquín utilizando el polvillo de caucho. La metodología fue cuantitativa y cualitativa puesto que la investigación realizo el análisis de las propiedades del caucho como elemento de construcción y de igual forma se realizaron encuestas sobre el conocimiento acerca del tema en la población utilizando encuentras entre otros métodos. Los resultados analizados fueron correlacionados con las propiedades de los adoquines convencionales donde se analizó el costo de elaboración por unidad y además se obtuvo la información sobre los porcentajes ideales de caucho obteniendo un resultado óptimo entre el 8 y 12 por ciento, también se obtuvo como resultado que mientras el porcentaje de caucho aumentaba su resistencia a la tracción indirecta disminuye.

Este trabajo de investigación tiene una relación con la investigación llevada a cabo ya que se investiga sobre las propiedades de los adoquines construidos con polvo de caucho sustituyendo el material fino también, tiene sus objetivos claros y descripción detallada [1].

\section{Bases teóricas}

Puesto que la investigación se enfoca en el caucho reciclado como material de construcción para la elaboración de un adoquín es de gran importancia mencionar y exponer apuntes, teorías y conceptos distintos con el fin de complementar y desarrollar la investigación de una manera óptima.

En primer lugar es de suma importancia tener en cuenta el concepto de adoquín, su funcionalidad, características físicas y visuales entre otras cosas para el desarrollo de esta investigación ya que el adoquín es un tipo de bloque usado en la construcción de pavimentos o embellecer sitios, como definición el adoquín es una piedra o bloque labrado de diferentes formas utilizado en la construcción de vías como pavimento [9]. Por otro lado también 


\section{8}

es definido como una unidad de concreto premezclado y vibro compactado de forma prismática cuyo diseño permite la colocación de forma continua y simétrica [10].

Como se aprecia en ambas definiciones la similitud es alta aunque el contexto es distinto, por un lado en la definición inicial se da una idea más abierta sobre los adoquines pues nos permite suponer que su forma y material puede variar al igual que su función por otro lado la siguiente descripción nos da una idea más centrada en el concreto como material de un adoquín y su simetría en forma.

Uno de los principales puntos de estudio en la investigación es el caucho reciclado obtenido del triturado de neumáticos desechados como elemento constructivo, para esto debemos saber la definición de caucho y neumático. El caucho por definición se conoce como una sustancia elástica, impermeable y resistente utilizada en la construcción de neumáticos por otra parte el neumático por definición es una cubierta de caucho o goma junto con otros agregados como el acero, óxido de zinc, textil, azufre, aditivos entre otros con el fin de soportar altas temperaturas y gran fricción con el pavimento. Teniendo en cuenta lo anterior para el diseño de un adoquín de caucho reciclado, debemos estudiar estos factores de resistencia con el fin de demostrar la posibilidad del uso del caucho en dicho diseño.

De la misma manera debemos de conocer el comportamiento de cada material de la mezcla final del adoquín y que puede generar si el componente se usa en exceso o por el contrario en poca cantidad. Por ejemplo si el caucho es usado en exceso que podría generar en el comportamiento general de adoquín o por el contrario que pasaría si se usa muy poco, si bien el caucho soporta esfuerzos friccionantes y temperaturas altas no significa que utilizado como reemplazo de agregado fino o grueso mejore la capacidad del adoquín a responder ante estos esfuerzos [11].

\section{Materiales y métodos}

La investigación será de tipo cualitativa la cual se basa en entender e interpretarla realidad tal y como es entendida por los sujetos participantes en los contextos estudiados [8]. Puesto que en el estudio del caucho como elemento constructivo se compararán datos y resultados de laboratorios realizados en otros proyectos en donde se implementa el caucho reciclado como parte de la creación de elementos constructivos para así determinar los distintos parámetros de resistencia a fuerzas y factores externos.

Del mismo modo se evaluará la información y su cumpliendo con la norma y estándares de construcción en reemplazo de los agregados del concreto para determinar el porcentaje óptimo estimado a usar. La estructura de la investigación se dividirá en dos partes. En primer lugar se llevará a cabo la investigación del caucho y sus especificaciones. Se evaluará su comportamiento y características cuando son sometidos a distintos agentes y esfuerzos basados en investigaciones anteriores para obtener una información óptima de su capacidad como un material capaz de reemplazar los agregados finos o gruesos.

Conforme se obtuvo la información se analizarán los resultados obtenidos en dichos proyectos para llegar a la conclusión de continuar con la segunda parte la cual sería llevada a cabo solo si los resultados obtenidos en la primera fase son los óptimos y mínimos para las necesidades que debe cumplir un adoquín.

\section{Resultados}

Adoquines para pavimento articulado. Desde la antigüedad el pavimento articulado ha sido una de las superficies de rodamiento más 
utilizadas tanto para paso peatonales como vehiculares.

Los adoquines, arena de juntas y cama de arena generan un mecanismo de trabazón mecánica entre los adoquines mediante el cual son capaces de disipar tensiones, transmitiendo carga entre adoquines adyacentes [12] describen el mecanismo de trabazón mecánica como el resultado del efecto combinado de cuña y rotación que se produce entre adoquines al ser cargados. En el efecto de cuña el adoquín cargado empuja longitudinalmente los adoquines vecinos.

El efecto de rotación en tanto, hace girar los adoquines vecinos en torno a alguno o varios de sus ejes. En adoquines rectangulares se produce el efecto de cuña o el efecto combinado de cuña y rotación en el caso de adoquines de caras dentadas. A lo anterior se agrega el aporte de la fricción entre las caras laterales de los adoquines, inducido por la arena en las juntas. Otro aspecto particular de los pavimentos de adoquines es que la trabazón mecánica aumenta con la aplicación de cargas verticales hasta llegar a un nivel de equilibrio. Este se alcanza aproximadamente después de 10.000 repeticiones de carga de un eje estándar (ejes equivalentes acumulados, EEA) [13]. Este comportamiento tiene asociado un aumento en la rigidez de la carpeta de rodadura, con lo cual aumenta su capacidad de disipar tensiones y consecuentemente disminuyen las tensiones que se transmiten a las capas inferiores del pavimento. Según [14] verificaron este aumento de la rigidez a través de mediciones en tramos de prueba y lo asociaron al comportamiento de las deformaciones permanentes del pavimento. Propusieron un modelo de progresión de las deformaciones permanentes en función de EEA de $80 \mathrm{kN}$.

Caucho reciclado en la construcción. El proceso de reciclaje de neumáticos es puramente mecánico, por tanto los productos resultantes son de alta calidad y limpios de todo tipo de impurezas, lo que facilita la reutilización de estos materiales en nuevos procesos y aplicaciones. La trituración con sistemas mecánicos es, casi siempre el paso previo en los diferentes métodos de recuperación y rentabilización de los residuos de neumáticos. Del tratamiento de los neumáticos fuera de uso se obtiene aproximadamente:

- $65 \%$ de caucho

- $15-25 \%$ de acero

$-10-15 \%$ de fibras textiles

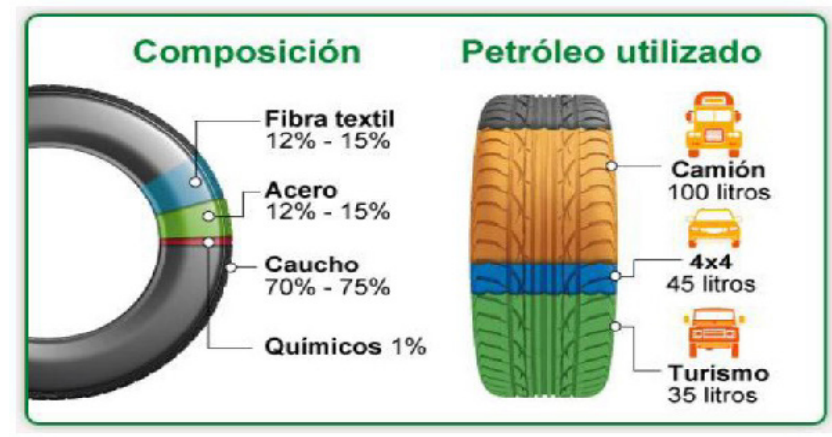

Composición y consumo petrolífero de neumáticos. [15]

Separando los restos aprovechables en la industria, el material resultante puede ser usado como parte de los componentes de las capas asfálticas que se usan en la construcción de carreteras, con lo que se consigue disminuir la extracción de áridos en carreteras.

Las carreteras con este tipo de asfalto son mejores y más seguras.Puede también usarse en aislantes de vehículos, losetas de goma, cubiertas, suelos de atletismo, suelas de zapato. Las utilidades son infinitas y cada día son más.Por ende el funcionamiento de caucho ha reemplazado de manera óptima los diferentes materiales en diferentes productos [14]. 


\section{0}

El caucho como elemento constructivo. Para analizar el caucho o neumático como elemento constructivo primero debemos de conocer sus propiedades, elementos y cómo se deben reciclar para su uso en la construcción. Un neumático es básicamente un elemento que permite a un vehículo desplazarse de forma suave atreves de las superficies. Consiste en una cubierta principalmente formada por caucho la cual contiene aire y el cual soporta al vehículo y su carga.

Un neumático obtiene su resistencia y solides por medio del proceso de vulcanización el cual consiste en recudir al mínimo sus propiedades plásticas para aumentar al máximo sus propiedades elásticas [16]. Puede ser $100 \%$ reciclable, sus componentes pueden ser reutilizados en diversas formas. Este proceso de extracción no es contaminante y consiste en separar de forma selectiva dichos componentes para su reutilización. Están compuestos por distintos materiales entre los cuales se encuentran: Caucho, acero, fibras textiles y químicos; estos materiales dotan de diferentes propiedades al neumático para que este pueda soportar su uso.

Las principales componentes y propiedades de los neumáticos son:

- Caucho: tiene excelentes propiedades mecánicas de tracción, flexión y compresión.

- Acero: mantener la rigidez ante los esfuerzos de carga.

- Fibras: material de gran poder calorífico con buenas propiedades de aislamiento acústico y termino [15].

Estas propiedades permiten el funcionamientos optimo del neumático ya que cada componente cumple una función específica y al trabajar en conjunto se logra que el neumático cumpla su función principal la cual es mantener el rodamiento suave y adherencia óptima.
Estos componentes resultan ser óptimos en el reciclaje pues al ser separados el material resultante puede ser usado como parte de los componentes de las capas asfálticas que se usan en la construcción de carreteras, con lo que se consigue disminuir la extracción de áridos en canteras.

Las carreteras que usan estos asfaltos son mejores y más seguras. Pueden usarse también en alfombras, aislantes de vehículos o losetas de goma, se han usado para materiales de pasos a nivel, cubiertas, masillas, aislantes de vibración. Las utilidades son infinitas y crecen cada día, como en cables de freno, compuestos de goma, suelas de zapato, bandas de retención de tráfico, compuestos para navegación o modificaciones del betún [17].

En la construcción el caucho o neumático se ha utilizado en numerosos espacios y ámbitos de la construcción, como se mencionó anteriormente la caucho ha sido utilizado en los caminos asfalticos, cubiertas entre otros casos y de la misma forma pueden ser utilizados como:

- Taludes de baja altura y Angulo: soportar el movimiento del terreno manteniéndolo de forma confinada.

- Campos de juego y pistas de paseo: crear una superficie más suave para que sea más cómodo al usuario.

- Juntas: mantener la separación entre juntas de edificaciones y disminuir el traspaso de energía durante movimientos telúricos.

Adoquines hechos a base de caucho reciclado Como hemos visto se les da una variedad de usos. Pero no solo se limitan a reutilizarlo Como un material compacto. El reciclado del neumático se ha llevado en otro nivel, es decirse ha explorado de una manera tal que 
a través de la separación de sus componentes se vuelven a darle otros usos, siendo el caucho y la fibra los más importantes para generar un nuevo producto.

Ladrillo ecológico de caucho. La exploración de este nuevo material surge como idea a través de una investigación llevada a cabo en Chile como [18].
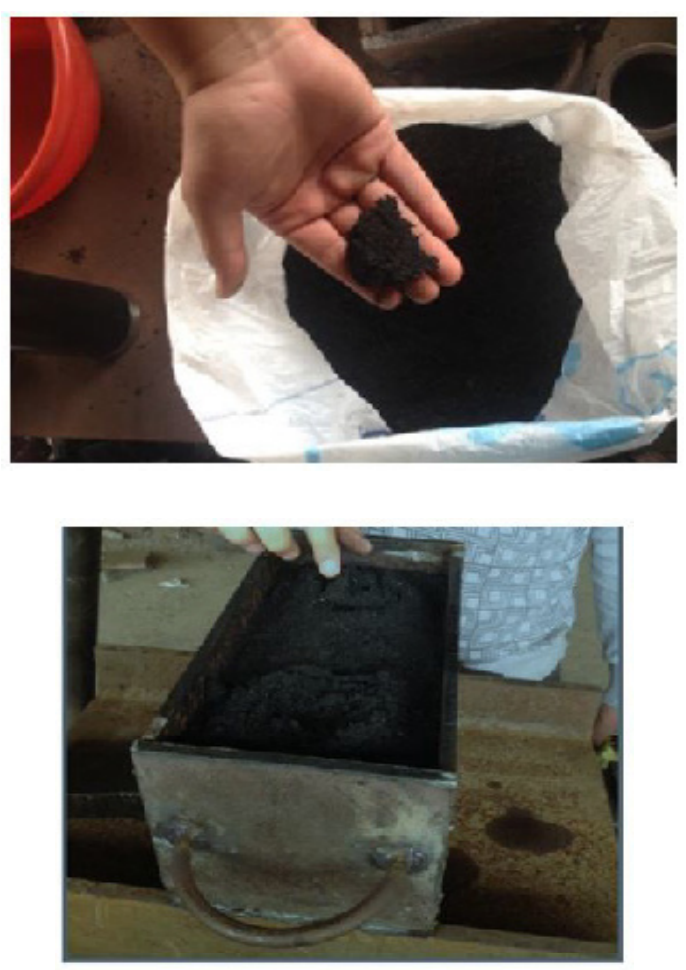

Molde

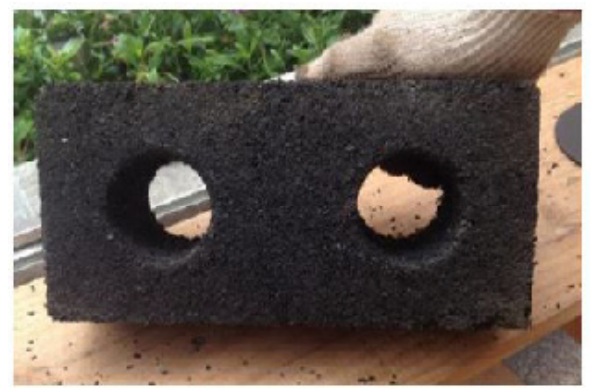

Ladrillo de caucho reciclado resultado final

Luego de haber realizado las pruebas de resistencia del material concluye que el mismo reúne los estándares de resistencia, comparando el mismo con los estándares de resistencia de un ladrillo cerámico.
Ventajas del material. Tiene una gran capacidad de impermeabilidad. Aislamiento acústico: no solo es un buen aislante acústico, sino que es mucho mejor aislante que un ladrillo cerámico, según las pruebas realizadas.

Desventajas del material. Debido a que está hecho de caucho (material altamente inflamable) el material es consumido por completo por las llamas. Siendo este punto un motivo por el que mejorar las características del material.

Con este trabajo de investigación ya están hechas los primeros pasos para ir explorando más acerca del caucho para la fabricación de ladrillos ecológicos, y en vista a ello, mejorar las características en las cuales faltaría y adoptarlo a nuestras características físicoambiental para que sea de gran utilidad y tener una forma de resolver el gran problema medioambiental que representa hoy día en nuestro país, el problema de los neumáticos fuera de uso.

Analisis y dosificacion estimada para un adoquín. Para la construcción adoquín básicamente se rige por tener una forma de tipo lego, y resistir al menos 3500 psi ya sea para caminos peatonales o vías vehiculares [19].

Basado en tesis anteriores por [20], según los estudios realizados y laboratorios demostrados en dichas tesis se ha llegado a la conclusión que para tener un funcionamiento óptimo de una estructura rígida a base de caucho reciclado se estima una cantidad entre el $15 \mathrm{y}$ $23 \%$ de la totalidad del adoquín.

Aspectos analizados para dicha dosificación estimada

- Al estar un material de solo caucho expuesto al exterior y sobre todo en clima caliente 


\section{2}

presentaba partes blandas no aptas para resistir cargas.

- Cuando el caucho era mezclado con otros agentes comunes en la construcción de elementos rígidos tenía un mejor comportamiento y mayor durabilidad a comparación del producto tradicional.

- El porcentaje fue entre el 15 y $23 \%$ debido a que estos eran en su mayoría los porcentajes más utilizados para ladrillos y asfaltos los cuales cumplían con la norma y resistencia requerida [20].

\section{Conclusiones}

Los desechos de neumáticos son enteramente reutilizables para la creación de diferentes productos no solo en la construcción sino en diferentes ámbitos. El caucho complementa de forma óptima materiales y a su vez otorga mejor resistencia y durabilidad a estos.

Con base en esta investigación se forma el inicio para explorar mas acerca del caucho para la fabricación de adoquines y mejorar las características de las cuales faltarían adoptar como estudios prácticos.

\section{Referencias}

[1] G. Wilson, History. of Solid Waste Management. Handbook of Salid Waste Management, New York: Editorial Van Nostrand, 1977

[2] G.J. Peláez, S.M. Velásquez y D.H. Giraldo, "Aditivos para el procesamiento del caucho natural y su aplicación en pequeñas plantaciones de caucho", trabajo de grado, Servicio Nacional de Aprendizaje -SENA, 2014.

[3] L.J. Franco Antolinez, M.A. Meza Joya y J.E. Almeira, "Situación de la disposición final de residuos sólidos en el Área Metropolitana de Bucaramanga: caso relleno sanitario El
Carrasco (revisión)", Avances: Investigación en ingeniería, vol. 15, no. 1, pp. 180-193, 2018

[4] G. Castro, "Materiales y compuesto para la industria del neumático", Trabajo de investigación de la F.I.U.B.A, Facultad de ingeniería de la universidad de Buenos Aires, 2008.

[5] La Opinión, "Cúcuta recicla solo el 6 por ciento de su basura", 2018. [En línea]. Disponible en: https://www.laopinion.com.co/cucuta/cucutarecicla-solo-el-6-por-ciento-de-su-basura154698\#OP

[6] A. Sarabia-Guarin, J. Sánchez-Molina, y J. C. Leyva-Díaz, "Uso de nutrientes tecnológicos como materia prima en la fabricación de materiales de construcción en el paradigma de la economía circular", Respuestas, vol. 22, no. 1, pp. 6 - 16, ene. 2017

[7] X.E. Castells, Libro reciclaje de residuos industriales, Madrid: Ediciones Díaz de Santos, 2000

[8] C.A Lasso Molina y A.E. Yugsi Toapanta, "Análisis de las propiedades mecánicas de adoquines elaborados con hormigón y polvillo de caucho de neumáticos reciclados y su correlación con adoquines convencionales", trabajo de grado, Universidad Central del Ecuador, 2018

[9] G. Peláez, S. Velásquez y D. Giraldo, "Aditivos para el Procesamiento del Caucho Natural y su Aplicación en Pequeñas Plantaciones de Caucho, 2014, http://revistas.sena.edu.co/index. php/inf_tec/article/view/100

[10] Periódico El Colombiano, 2015, https:// www.elcolombiano.com/colombia/basuras-yrellenos-sanitarios-problematica-en-colombiaHB7636867 
[11] Periódico El Tiempo octubre 24 de 2016, https:// www.eltiempo.com/vida/ciencia/reciclaje-de1lantas-en-colombia-52722

[12] R. Gregorio, 1999. [En línea]. Disponible en: https://www.santafe.com.co/

[13] S.G. Quevedo Rivera, C.A. Guamán, "Altamirano, Proyecto de factibilidad para la producción de Eco-adoquines peatonales mediante la reutilización de desechos plásticos (PET)", trabajo de grado, Escuela Superior Politécnica Superior de Chimborazo, 2013

[14] Adoquin Ecológico, 1986. [En línea]. Disponible en: http://adoquinecologico. blogspot.com/2017/11/adoquin-ecologico_27. html

[15] Definición de adoquin, 2015. [En línea]. Disponible en: https://www.definiciones-de. com/Definicion/de/adoquin.php

[16] Ventajas del adoquin ecológico, 1990. [En línea]. Disponible en: http://ecotelhado.com.co/ ventajas-del-adoquin-ecologico/

[17] Arcillas Amaya, 2011. [En línea]. Disponible en: https://www.arcillasamaya.com/

[18] G.J. Peláez Arroyave, S.M. Velásquez Restrepo y D.H. Giraldo Vásquez, "Aplicaciones de caucho reciclado: Una revisión de la literatura", Ciencia e Ingeniería Neogranadina, vol. 27, no. 2, 2017

[19] Adoquines Colombia, 2010. [En línea]. Disponible en: https://www.planetacolombia. com/cucuta/adoquines

[20] A. Alfonzo Ortega, Eco-ladrillo a base de caucho reciclado de neumáticos fuera de uso, trabajo de grado, Universidad Nacional de Asunción, 2016 